\title{
Central Obesity and BMI Associated with Different Phenotypes of Polycystic Ovary Syndrome in Adolescent
}

\author{
Bahar Morshed Behbahani ${ }^{1}$, Marzieh Akbaezadeh ${ }^{2}$ \\ ${ }^{1}$ Department of Midwifery, School of Nursing and Midwifery, Shiraz University of Medical Sciences, Iran \\ ${ }^{2}$ Community Based Psychiatric Care Research Center, Department of Midwifery, Fatemeh (P.B.U.H) School \\ of Nursing and Midwifery, Shiraz University of Medical Sciences, Iran
}

\begin{abstract}
Article Info
Article history:

Received Sep 15, 2017

Revised Nov 10, 2017

Accepted Nov 29, 2017

\section{Keyword:}

Android obesity

BMI

Phenotypes

PCOS

Teenagers

ABSTRACT

Adipose tissue distribution is effective in metabolic complications resulting from obesity. Fat accumulation in the body, particularly increase in visceral fat, is a major risk factor of diabetes and cardiovascular diseases. The present study aimed to determine the prevalence of obesity and android obesity based on various phenotypes of Polycystic Ovarian Syndrome (PCOS) in 14-18 year old high-school girl students in Shiraz in 2009. This descriptive study was conducted on 14-18 year old high-school girl students in Shiraz in 2009. Considering the confidence coefficient of $95 \%$ and loss rate of $20 \%$, a 3190 subject sample size was determined for the study. The participants were selected through purposive sampling. After obtaining written informed consents, demographic information questionnaire was completed and the participants were examined regarding hirsutism, acne, alopecia, and menstrual disorders. Besides, the criteria proposed by Adams et al. were employed to diagnose PCOS in sonography. Android obesity and Body Mass Index (BMI) were evaluated, as well. The results of independent t-test showed no significant difference among various phenotypes of PCOS regarding mean android obesity and BMI $(\mathrm{P}>0.05)$. The results of the present study revealed no significant relationship between girl adolescents' obesity and various phenotypes of PCOS. However, adolescents should be informed about the long-term hyperandrogenic outcomes which are accompanied by insulin secretion and endanger their health after the age of 40 years.
\end{abstract}

Copyright $@ 2017$ Institute of Advanced Engineering and Science. All rights reserved.

Corresponding Author:

Bahar Morshed Behbahani

Department of Midwifery, School of Nursing and Midwifery,

Shiraz University of Medical Sciences, Shiraz, Islamic Republic of Iran.

Email: morshe_b@yahoo.com

\section{INTRODUCTION}

In Europe, Polycystic Ovarian Syndrome (PCOS) is diagnosed by existence of cystic ovaries in sonography together with oligomenorrhea, hyperandrogenism, obesity, or increase of serum LH or testosterone. In the U.S., on the other hand, National Institute Health (NIH) in 1990 suggested hyperandrogenism or hyperandrogenemia and ovulatory dysfunction in the absence of non-classical adrenal hyperplasia as the diagnostic criteria for PCOS [1]. PCOS is mainly accompanied by oligomenorrhea or amenorrhea and is identified by the clinical or laboratory indications of hyperandrogenemia. Therefore, the prevalence rate of PCOS is varied from $6 \%$ to $22 \%$ in different parts of the world [2],[3] and the difference in statistics results from different criteria used for diagnosis of this syndrome [4]. Nowadays, it has been shown that a large number of obese women with PCOS suffer from hyperinsulinemia, as well [5]. Moreover, many adolescents suffering from PCOS are overweight or obese. It seems that PCOS is the endocrine cause of 
obesity in girl adolescents [6]. Recent systematic studies have shown that PCOS can result from the same genetic factors which lead to obesity in women. Yet, it is not known whether obesity is the primary factor in the incidence of PCOS or vice versa. Overall, the women with PCOS are more obese abdominally compared to those with similar weight and Body Mass Index (BMI) [7]. Even non-obese PCOS adolescents have twice as much abdominal fat as the general population [8]. Android obesity is highly correlated to hyperandrogenism and BMI is correlated to insulin resistance [9].

The results of the study by Trent (2002) showed that weight problems caused stress and anxiety for the individuals suffering from PCOS. Hence, psychological problems and difficulty in determining one's health and identity is another important dimension of this syndrome. Evidence has shown that adult females with PCOS normally suffer from infertility and its outcomes, while such adolescents are more concerned with weight gain, menstrual disorders, acne, and hirsutism. These complications seriously affect young girls' quality of life. Therefore, young adults suffering from this syndrome must be diagnosed and treated as soon as possible.

\section{MATERIALS AND METHODS}

This descriptive study was conducted on 14-18 year old high-school girl students in Shiraz in 2009. Based on a similar study performed in Isfahan [10] and considering the confidence coefficient of $95 \%$ and loss rate of $20 \%$, a 3190-subject sample size was determined for the study. Then, 600-800 students were selected from each educational district using purposive sampling. The inclusion criteria of the study were being 14-18 years old and not consuming any hormones and medications except for anti-allergy and analgesic drugs. On the other hand, the exclusion criteria of the study were passage of less than 2 years from menarche, having adrenal or thyroid problems, suffering from hyperprolactinemia, primary amenorrhea, serious underlying diseases, such as malignancies and thalassemia, which affect menstrual cycles, and having identified endocrinopathies, such as Cushing syndrome.

After obtaining written informed consents, demographic information questionnaire was completed and the participants were examined regarding hirsutism, acne, alopecia, and menstrual disorders. Besides, the criteria proposed by Adams et al. were utilized to diagnose PCOS in sonography [11]. The participants' waist circumference was measured as the distance between the last rib and the iliac crest at the end of expiration in standing position. In order to determine android obesity, based on waist circumference and age, percentiles $>90$ were considered as android obesity [12],[13].

In order to compute BMI, at first, the participants' height was measured without shoes using a tape meter installed on the wall $40 \mathrm{~cm}$ higher than the ground level. In doing so, the participants were asked to stick their back of head, waist, hip, and feet to the wall and their height was determined by placing a ruler on their head. Then, the participants' weight was measured barefoot with the least amount of clothing using CAMRY scale (made in China) which was previously adjusted by standard weights [14].

BMI charts provided by the Center for Disease Control (CDC) were used to determine underweight, overweight, and obesity. Accordingly, 85-95 percentiles were considered as overweight, while percentiles $\geq 95$ were considered as obese [12]. After all, the data were entered into the SPSS statistical software (version $11.5)$ and analyzed using Chi-square test.

\section{RESULTS}

Out of the 41 government high schools in Shiraz, 16 ones took part in the present study. The study was conducted on 3190 high-school girl students between 14 and 18 years old in Shiraz. According to the results, most of the participants $(30.2 \%)$ were 17 years old. The participants' mean weight, mean height, and mean waist circumference were $54.14 \pm 9.99 \mathrm{~kg}, 160.20 \pm 5.76 \mathrm{~cm}$, and $65.24 \pm 7.34 \mathrm{~cm}$, respectively. In addition, 616 participants $(20.5 \%)$ were underweight, 234 ones $(9.5 \%)$ were overweight, and 110 ones $(3.5 \%)$ were obese. Also, 15 participants $(0.5 \%)$ had android obesity.

The results of independent t-test revealed no significant difference between the phenotypes of the participants with hyperandrogenism and PCOS and those without these disorders, phenotypes of the participants with oligomenorrhea and PCOS and those not suffering from these disorders $(\mathrm{P}=0.41)$, and phenotypes of the participants with oligomenorrhea, PCOS, and hyperandrogenims and those without these disorders concerning the mean of android obesity and BMI $(\mathrm{P}=0.5)$.

Overall, $16.6 \%$ of the study participants (514 subjects) suffered from menstrual disorders and oligomenorrhea. Also, 99 participants $(3.1 \%)$ had $\mathrm{BMI} \geq 26$. 


\section{DISCUSSIONS}

The results of the present study showed that the prevalence rates of overweight and obesity in Shiraz were similar to those reported in developed countries and other parts of Iran. For instance, Salem et al. (2007) conducted a study on 11-18 year old girls in Rafsanjan and reported the prevalence rates of overweight and obesity to be $11.2 \%$ and $2.4 \%$, respectively [15]. In the present study, $9.5 \%$ of the participants were overweight and $3.5 \%$ were obese. One study performed in the U.S. also showed that $16.8 \%$ of 2-19 year old girls were obese [16]. In another study conducted in Greece, the prevalence rates of overweight and obesity were $19 \%$ and $2.6 \%$, respectively among 11-17 year old adolescents and these rates were higher among males compared to females [17]. The prevalence of obesity is also high in developing countries. In India, for example, the prevalence rates of overweight and obesity among 9-15 year old children have been reported to be $11.1 \%$ and $14.2 \%$, respectively [18]. Hekimsoy (2003) mentioned obesity as an important risk factor of type II diabetes, arterial hypertension, and hyperlipidemia. However, contradictory results have been obtained regarding the relationship between duration of obesity and these risks [19]. Thus, obesity has been considered as a major problem for girls' health in future.

In the current study, the prevalence of android obesity was $0.5 \%$ which was lower compared to the measures obtained in the studies performed by Salem and Halley Castillo (2007) [20]. In spite of the fact that the prevalence rate of android obesity was low in this study, it is still an alarming issue because according to the studies conducted in Asia, android obesity can increase the risk of PCOS by two folds (RR=2.41, P=0.04) [4]. It can also increase the incidence rate of metabolic syndrome in future. In fact, android obesity leads to resistance in consumption of peripheral glucose and results in hypertension and further metabolic problems by causing hyperinsulinemia [21]. The low prevalence of android obesity in the present study might be due to the participants' age and sex. In fact, female teenagers are highly concerned about android obesity.

The results of this study also indicated that the prevalence rates of obesity and android obesity were higher in some phenotypes of PCOS. However, no significant difference was observed between the PCOS patients and those not suffering from this syndrome in this regard. Of course, this was not unexpected because based on the recent systematic review studies conducted on this issue; Asian women are less obese compared to the Caucasian women suffering from PCOS [4]. Thus, the incidence of PCOS might also be associated with racial factors. Yet, other studies have indicated that almost $25-50 \%$ of the women with PCOS are obese [22],[23]. These measures have been reported as 10-65\% in some studies. Also, a large number of adolescents suffering from PCOS are either overweight or obese. In fact, PCOS is the most common endocrine cause of obesity in adolescents [24]. In one study, the prevalence of obesity was reported as $27 \%$ among the adolescents with PCOS [25].

The findings of the present study revealed no significant relationship between BMI and menstrual patterns. In this study, out of the $16.6 \%$ of the participants with menstrual disorders, $3.1 \%$ (99) were overweight or obese. Evidence has shown that the individuals with oligomenorrhea have higher BMI. Sarmeli et al. (2002) also reported high BMI in 19 girls between 16 and 18 years old who suffered from oligomenorrhea. Since obesity increases the androgen levels, it is usually accompanied by PCOS and oligomenorrhea. Furthermore, irregular and long menstrual cycles might have genetic background, as well. Nevertheless, the difference between the results of the present study and those of other studies conducted on the issue might be due to the adolescents' nutritional status and their great concern for appropriate waist and hip circumference. Of course, since individuals genetically have the disorder, its symptoms are intensified by environmental factors and individuals' lifestyles. BMI is one of the main environmental factors which can intensify or reduce the severity of the disorder [26]-[29].

\section{CONCLUSION}

The results of the present study revealed no significant difference between Iranian girl adolescents and those in developed countries regarding the prevalence of obesity. This is an alarming issue for the related health organizations. Thus, appropriate educational and social strategies should be suggested for preventing this problem, because overweight can lead to metabolic syndrome and PCOS which are serious threats for health. Moreover, adolescents should be informed about the long-term hyperandrogenic outcomes which are accompanied by insulin secretion and endanger their health after the age of 40 years. The study findings also indicated that various phenotypes of PCOS in adolescents were not significantly related to obesity and overweight. Therefore, further studies are required to investigate the psychological and genetic causes of the incidence of this syndrome. 


\section{REFERENCES}

[1] Balen A. H., et al., "Polycystic ovary syndrome.the spectrum of the disorder in 1741 patients," Hum Reprod., vol/issue: 10(8), pp. 2107-11, 1995.

[2] Asunción M., et al., "A prospective study of the prevalence of the polycystic ovary syndrome in unselected Caucasian women from Spain," J Clin Endocrinol Metab., vol/issue: 85(4), pp. 1735-40, 2000.

[3] D. Kandarakis E., et al., "A survey of the polycystic ovary syndrome in the Greek island of Lesbos: hormonal and metabolic profile,” J Clin Endocrinol Metab., vol/issue: 84(11), pp. 4006-11, 1999.

[4] B. O. Yildiz, et al., "Prevalence, phenotype and cardiometabolic risk of polycystic ovary syndrome under different diagnostic criteria," Human Reproduction, vol/issue: 27(10), pp. 3067-3073, 2012.

[5] B. Janatans, "Novake's Gynecology," $13^{\text {th }}$ ed. Lippincott Williams \& Wikins, pp. 876-880, 2005.

[6] In-Iw S. and Biro F. M., “Adolescent women and obesity,” J Pediatr Adolesc Gynecol., vol/issue: 24(2), pp. 58-61, 2011.

[7] S. S. Lim, et al., "Overweight, obesity and central obesity in women with polycystic ovary syndrome: a systematic review and meta-analysis," Human Reproduction Update, vol/issue: 18(6), pp. 618-637, 2012.

[8] Ibanes L. and de Zegher F., "Ethinylesttradiol - drospirenone, flutamide - metformin, or both for adolescents and women with hyperinsulinemia hyperandrogenism: opposite effects on adipocytokines and body adiposity," The Journal of clinical Endocrinology and Metabolism, vol/issue: 89(4), pp. 1592 -1597, 2004.

[9] Wild R. A., "Obesity, lipid, cardiovascular risk and androgen excess," The American Journal of Medicine, vol/issue: 98(1A), pp. 275-325, 1995.

[10] Plouffe Jr. L., "Disorders of excessive hair growth in the adolescent," Obtetrics and Gynecology Clinics of North America, vol/issue: 27(1), pp. 79, 2000.

[11] Adams J., et al., "Prevalence of polycystic ovaries in women with idiopathic hirsutism," Br Med J Clin Res Ed., vol. 293, pp. 355-9, 1986

[12] Malekzadeh R., et al., "Obesity pandemic: An Iranian perspective," Arch Iran Med., vol/issue: 8(1), pp. 1-7, 2006.

[13] Dunaif A., et al., "Profound peripheral insulin resistance, independent of obesity, in polycystic ovary syndrome," Diabetes, vol/issue: 38(9), pp. 1165-1174, 1989.

[14] Duncan G. E., et al., "Prevalence and trends of metabolic syndrom Phenotype among U.S adolescents, 1999-2000," Diabetes Care, vol/issue: 27(10), pp. 2438-43, 2004.

[15] Salem Z. and Vazirinejad R., "Prevalence of obesity and metabolic syndrome in girls aged 11-18 years in Rafsenjan city," Journal Diabetes and Lipid of Iran, vol/issue: 7(2), pp. 205-213, 1986.

[16] Ogden C. L., et al., "Prevalence of obesity and trends in body mass index among US children and adolescents, 1999-2010," JAMA, vol/issue: 307(5), pp. 483-90, 2012.

[17] Krassas G. E., et al., "Prevalence and trenda in overweight and obesity among children and adolescents in Thessaloniki, Greece," J Pediatr Endocrinol Metab., vol/issue: 14(5), pp. 1319-26, 2001.

[18] Chhatwal J., et al., "Obesity among pre-adolescent and adolescents of a developing country (India)," Asia Pac J Clin Nutt., vol/issue: 13(3), pp. 231-5, 2004.

[19] Hekimsoy Z. and Oktern I. K., "Duration of obesity is not a risk factor for type 2 diabete mellitus, arterial hypertention and hyperlipidemia," Diabetes, Obesity and Metabolism, vol/issue: 5(5), pp. 432, 2003.

[20] H. Castillo E., et al., "Body mass index and the prevalence of metabolic syndrome among children and adolescents in two Mexican population," J Adolesc Healt, vol/issue: 40(6), pp. 521-6, 2007.

[21] Pyorala M., et al., "Hyperinsulinemia predicates coronary heart disease risk in healt middle aged men. The 22 year follow-up results of the Helsinki policmen study," Circulation, vol/issue: 98(5), pp. 398-404, 1998.

[22] Buggc C. and Rosenfield R. L., "Polycystic ovary stndrome in adolescence," Endocrinol Metab Clin North Am., vol/issue: 34(3), pp. 677-705, 2005.

[23] Azziz R., "Androgen excess is the key element in polycystic ovary syndrome," Fertil Steril, vol/issue: 80(2), pp. 252-4, 2003.

[24] Christopher R., et al., "The Association of obesity and hyperandrogenemia during the pubertal Transition in girls," The Journal of Clinical Endocrinology \& Metabolism, vol/issue: 91(5), pp. 1714-1722, 2005.

[25] Dramusic V., et al., "Clinical, endocrinologic, and ultrasonograpfic features of polycystic ovary syndrome in Singaporean adolescents," Journal of Pediatric and Adolescent Gynecology, vol/issue: 10(3), pp. 125-132, 1997.

[26] H. Teede, et al., "Cystic ovary syndrome: a complex condition with psychological, reproductive and metabolic manifestations that impacts on health across the lifespan," BMC Medicine, vol. 8, pp. 41-51, 2010.

[27] Balen A. H., et al., "Polycystic ovary syndrome: the spectrum of the disorder in 1741 patients," Hum Reprod., vol/issue: 10(8), pp. 2107-11, 1995.

[28] Homburg R. and Lambalk C. B., "Polycystic ovary syndrome in adolescence - a therapeutic conundrum," Human Reproduction, vol/issue: 19(5), pp. 1039-1042, 2004.

[29] Csermely T., et al., "Occurrence of osteopenia among adolescent girlswith oligoamenorrhea," Gynecol Endoerinal, vol/issue: 16(2), pp. 99-105, 2002. 